Chirurg $2021 \cdot 92: 916-917$

https://doi.org/10.1007/s00104-021-01504-8

Angenommen: 16. August 2021

(c) Springer Medizin Verlag GmbH, ein Teil von Springer Nature 2021

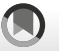

\section{Auswirkung von COVID-19 in der Viszeralchirurgie}

Christiane J. Bruns

Klinik und Poliklinik für Allgemein-, Viszeral-, Tumor- und Transplantationschirurgie, Universitätsklinikum Köln (AöR), Köln, Deutschland
Die COVID-19-Pandemie hat über das gesamte Jahr 2020 das private und berufliche Leben der Menschen weltweit verändert. Auch im Jahr 2021 bleiben diese Lebensveränderungen teilweise bestehen.

Das Gesundheitssystem war 2020 und ist sicher auch noch einige Jahre weiter in allen Bereichen von der Hausarztpraxis bis zum universitären Maximalversorger transsektoral extrem gefordert, einerseits die Pandemie zu beherrschen, andererseits die generelle Gesundheitsversorgung der Bevölkerung vollumfänglich sicherzustellen. So mussten in den ersten 12 Wochen der Pandemie weltweit bereits über 30 Mio. Operationen bzw. $80 \%$ der elektiven nichtonkologischen Operationen verschoben werden (Lock et al.).

Neben den vielen mehr oder weniger schwer COVID-19-erkrankten Patienten, die stationäre bis hin zu intensivstationärer Behandlung benötigten, waren insbesondere solche Patienten betroffen, die aufgrund einer Non-COVID-Erkrankung mit mittel- bis langfristig lebensbedrohlichen Folgen eine komplexe, zumeist operative und intensivmedizinische Behandlung benötigten. Die Beiträge in dieser Ausgabe befassen sich mit den Auswirkungen der COVID-19-Pandemie auf die onkologische Chirurgie des oberen Gastrointestinaltrakts, die elektive und notfallmäßige Kolorektalchirurgie sowie die hepatopankreatobiliäre (HPB) Chirurgie und die Organtransplantation.

Mit Ausbruch der COVID-19-Pandemie kam es zu deutlichen Einschränkungen in der gesamten elektiven Chirurgie und den damit verbundenen Standards der jeweiligen Krankenhäuser. So sind gerade in der onkologischen Chirurgie zumeist therapeutische Zeitfenster bis zur Operation einzuhalten, entsprechend mussten die begrenzten Intensivkapazitäten kritisch verteilt werden, um sowohl Akutund COVID-19-Erkrankten als auch Tumorpatienten gerecht zu werden. Babic et al. beschreiben die Auswirkungen der COVID-19-Pandemie auf die onkologische Ösophaguschirurgie. Weitestgehend im Einklang mit internationalen Daten wich die anfängliche Zurückhaltung bezüglich elektiver Zweihöhleneingriffe und dabei insbesondere minimal-invasiver Chirurgie mit zunehmendem Wissensstand über die COVID-19-Pandemie, sodass insgesamt onkologische Eingriffe des oberen GI-Traktes (Gastrointestinaltrakt) über das Jahr 2020 mit einem hohen Maß an Sicherheit und in der Regel internationalen Leitlinien entsprechend durchgeführt werden konnten.

\section{I) Mit Ausbruch der COVID-19- Pandemie kam es zu deutlichen Einschränkungen in der elektiven Chirurgie}

Neben der Verschiebung elektiver Operationstermine können aber auch eine verzögerte Diagnostik und Vorsorge insbesondere im Bereich von kolorektalen Karzinomen zu weitreichenden Folgen in der Prognose führen. Die kolorektale Chirurgie stellt nach ihrer Fallzahl die größte Subspezialität in der Viszeralchirurgie dar, wobei die chirurgische Therapie des kolorektalen Karzinoms sowie die operative Therapie abdomineller Infektionen wie Divertikulitis und Appendizitis am häufigsten sind. Lock et al. diskutieren aktuelle Empfehlungen zum perioperativen Management in Bezug auf SARS-CoV-2 und die möglichen kurz-und mittelfristigen Auswirkungen auf die kolorektale Chirurgie. Die SARS-CoV-2Pandemie hat zu erheblichen Veränderun- 
gen in der kolorektalen Chirurgie geführt und wird auch über die kommenden Jahre einen erheblichen Einfluss auf die chirurgische Versorgung der Patienten haben, da es speziell für das kolorektale Karzinom zu einer reduzierten Früherkennungsrate kommen wird, mehr Patienten in einem fortgeschrittenen Tumorstadium diagnostiziert werden und entsprechend in den nächsten 5 bis 10 Jahren eine deutliche Übersterblichkeit beim kolorektalen Karzinom auftreten wird.

Hillebrandt et al. beleuchten die direkten und indirekten Folgen der COVID-19Pandemie auf die hepatopankreatobiliäre (HPB) Chirurgie und Organtransplantation unter Berücksichtigung von aktueller Literatur und veröffentlichten Expertenmeinungen nationaler und internationaler Fachgesellschaften. Insbesondere in der ersten Pandemiewelle zeigte sich in den Bereichen der HPB-Chirurgie und Organtransplantation an vielen Zentren ein deutlicher Einbruch der Eingriffszahlen ohne kompensatorischen Zuwachs in den Folgemonaten. Ob dieser Trend auf eine erhöhte Sterblichkeit durch verschobene Operationen oder geänderte Therapieregime zurückzuführen ist, lässt sich derzeit zumindest nicht ausschließen.

Einerseits ist eine SARS-CoV-2-Infektion eher mit einem komplikativen perioperativen Verlauf bei diesen Patienten assoziiert, insbesondere nach Organtransplantation. Andererseits haben Patienten mit vornehmlich onkologischen Erkrankungen des hepatopankreatobiliären Systems oder im Rahmen einer Organtransplantation aufgrund eines komplexen Komorbiditätsprofils ein deutlich erhöhtes Risiko für einen schweren COVID-19-Verlauf. Dies muss bei der Abwägung von Therapiealternativen, den Schutzempfehlungen und der Priorisierung bei Impfungen in Betracht gezogen werden (Hillebrandt et al.).

Christiane J. Bruns

\section{Korrespondenzadresse}

Univ.-Prof. Dr. med. Christiane J. Bruns, FEBS

Klinik und Poliklinik für Allgemein-, Viszeral-, Tumor- und Transplantationschirurgie, Universitätsklinikum Köln (AöR) Kerpener Str. 62, 50937 Köln, Deutschland christiane.bruns@uk-koeln.de

Interessenkonflikt. C.J. Bruns gibt an, dass kein Interessenkonflikt besteht.
Hier steht eine Anzeige. Springer 